\title{
Stellar luminosity functions of rich star clusters in the Large Magellanic Cloud
}

\author{
B. Santiago ${ }^{1}$, S. Beaulieu ${ }^{2}$, R. Johnson ${ }^{2}$, and G. F. Gilmore ${ }^{2}$ \\ 1 Universidade Federal Rio Grande do Sul, IF, CP 15051, Porto Alegre 91501-970, RS, Brasil \\ 2 Institute of Astronomy, Madingley Rd., Cambridge CB3 0HA, UK
}

Received 8 September 2000 / Accepted 12 December 2000

\begin{abstract}
We show the results of deep $V$ and $I$ HST photometry of 6 rich star clusters in the Large Magellanic Cloud with different ages and metallicities. The number of stars with measured magnitudes in each cluster varies from about 3000 to 10000 stars. We build stellar density and surface brightness profiles for the clusters and extract half-light radii and other structural parameters for each. We also obtain luminosity functions, $\Phi\left(M_{V}\right)$, down to $M_{V} \simeq 6\left(M / M_{\odot} \gtrsim 0.9\right)$, and investigate their dependence with distance from the cluster centre well beyond their half-light radius. In all clusters we find a systematic increase in the luminosity functions slope, $\Delta \log \Phi\left(M_{V}\right) / \Delta\left(M_{V}\right)$, with radial distance from the centre. Among the clusters displaying significant mass segregation are the 2 youngest in the sample: NGC 1805, NGC 1818. For these two clusters we obtain present day mass functions. The NGC 1818 mass function is in excellent agreement with that derived by other authors, also using HST data. The young cluster mass function slopes differ, that of NGC 1805 being systematically steeper than NGC 1818. Since these are very young stellar systems ( $\tau \lesssim 40 \mathrm{Myrs}$ ), these variations may reflect the initial conditions rather than evolution due to internal dynamics.
\end{abstract}

Key words. stars: luminosity function, mass function; galaxies: clusters: general; Magellanic Clouds

\section{Introduction}

Rich star clusters are made up of tens and sometimes hundreds of thousands of stars bound together by gravity. Models for the formation and evolution of such systems have to incorporate effects of dynamical friction, stellar evaporation, tidal effects, disc shocks, twobody interactions and stellar evolution (Heggie \& Aarseth 1992; Spurzem \& Aarseth 1996; Vesperini \& Heggie 1997; Goodwin 1997a; Aarseth 1999). Substantial progress is being made towards understanding such dynamical effects and realistically modeling them. Model predictions generally make use of $N$-body simulations and can more easily be tested for a particular cluster (or set of clusters) if enough information about its present day structure, shape, density and velocity field is available for comparison (Vesperini \& Heggie 1997; Goodwin 1997b; de Oliveira et al. 2000). With $N$-body models one can also predict the luminosity function and mass function for a cluster at some distance from the centre and at some age. This process yields insight on the initial conditions under which the cluster(s) formed, i.e., the initial mass function

Send offprint requests to: B. Santiago,

e-mail: santiago@if.ufrgs.br
(IMF), density profile, amount of original stellar segregation, etc. The stellar IMF, in particular, is a very valuable piece of information as many fundamental astrophysical issues depend on its shape and possible universality (Chiosi et al. 1998).

Recovering initial conditions from the current properties of a cluster becomes harder the older it is, since more time has elapsed for internal dynamics and stellar evolution to erase them. Unfortunately, our Milky-Way galaxy contains a system of purely old (ages $\tau \gtrsim 10^{10}$ years) rich globular clusters, mostly located in the Galactic halo. The nearest galaxy with a diverse system of rich star clusters, spanning a wide range in ages, metallicities and positions is the Large Magellanic Cloud (LMC) (Westerlund 1990). It is therefore a suitable laboratory for studying the processes by which clusters form and evolve.

In this paper we describe the analysis of density profiles and luminosity functions of 6 rich LMC clusters, deliberately chosen so as to encompass a large range in age and metallicity. The results are intended as a useful observational input to modelling by $N$-body experiments (including those we have underway). The data come from Hubble Space Telescope (HST) observations using the Wide Field and Planetary Camera 2 (WFPC2). 
With the high-resolution imaging provided by this instrumental setup it was possible to obtain luminosity functions deeper than in any previous attempt. The paper is outlined as follows: in Sect. 2 we describe the data, the stellar samples derived from them, and the photometry. Density profiles and position-dependent luminosity functions are presented in Sect. 3. In Sect. 4 we discuss our results and compare them with other works. Finally, in Sect. 5 we present our conclusions.

\section{Observations}

These WFPC2 data are part of the cycle 7 HST 7307 project (Beaulieu et al. 1998; Elson et al. 1998a,b; Johnson et al. 1998). The project includes observations of 8 clusters: NGC 1805, NGC 1818, NGC 1831, NGC 1868, NGC 2209, Hodge 14, Hodge 11 and NGC 2210. The two latter are old systems $\left(\tau>10^{10}\right.$ years $)$ and therefore have a much fainter main-sequence turn-off than the others. As our main interest is to analyze the shape of main-sequence luminosity functions, we here concentrate on the remaining 6 clusters.

For each cluster, two sets of WFPC2 images were obtained, one with the centre of the Planetary Camera (PC) coinciding with the cluster's center and the other with the PC centered on the cluster's half-light radius. We call these the CEN and HALF fields, respectively. 6 exposures were obtained with each of the F814W and F555W filters in the CEN field: 3 exposures were deeper (individual exposure times of $300 \mathrm{~s}$ and $140 \mathrm{~s}$, respectively, for F814W and $\mathrm{F} 555 \mathrm{~W}$ ), the remaining 3 were shallow (20 s and $5 \mathrm{~s}$, respectively, for F814W and F555W); the shallower exposures were meant to allow for photometry of bright stars. For the HALF fields, 3 deep exposures (total exposure time: $2500 \mathrm{~s}$ ) were taken with each filter. Saturation for these latter was a problem only for $m_{555} \lesssim 19$.

WFPC2 images located about $7^{\prime}$ away from each cluster are also available as part of the 7307 project. These are parallel observations with NICMOS as the primary instrument. Two exposures were taken with each of the two filters, the total exposure time being $1200 \mathrm{~s}$ for F555W and $800 \mathrm{~s}$ for F $814 \mathrm{~W}$. These were used for subtraction of background field LMC stars. Colour-magnitude diagrams (CMDs) for the field stars in the images were studied by Castro et al. (2000).

The 3 individual exposures at each pointing, filter and exposure time were combined together. No registration was required. The same was done for the 2 exposures in the parallel fields used for field star subtraction. The final stacked image in each case was then used for sample selection and photometry.

In Table 1 we list the basic properties of the clusters plus the total number of stars found in our images. The data in the table were taken from Elson (1991), Corsi et al. (1994), Will et al. (1995), Mateo (1988) and Johnson et al. (2000).
Table 1. Cluster's parameters

\begin{tabular}{|c|c|c|c|c|c|}
\hline Cluster & $\theta_{\mathrm{LMC}}(\circ)$ & $E(B-V)$ & log Age (yrs) & {$[\mathrm{Fe} / \mathrm{H}]$} & $N_{\text {stars }}$ \\
\hline NGC 1805 & 4.1 & 0.04 & $7.0-7.3$ & -0.4 & 6508 \\
\hline NGC 1818 & 3.8 & 0.03 & $7.3-7.6$ & -0.4 & 10187 \\
\hline NGC 1831 & 5.1 & 0.05 & $8.5-8.7$ & -0.3 & 7801 \\
\hline NGC 1868 & 5.9 & 0.02 & 8.8 & -0.6 & 7362 \\
\hline NGC 2209 & 5.4 & 0.07 & $8.9-9.1$ & -1.0 & 3771 \\
\hline Hodge 14 & 3.9 & 0.04 & $9.2-9.4$ & -0.7 & 3133 \\
\hline
\end{tabular}

\subsection{Sample selection}

The IRAF DAOPHOT package was used to detect and classify sources automatically in each stacked F814W image. Star candidates were detected using DAOFIND, with a peak intensity threshold for detection set to $5 \sigma$, where $\sigma$ corresponds to the rms fluctuation in the sky counts, determined individually for each image.

A preliminary aperture photometry was carried out by running the task PHOT on all objects output by DAOFIND. Star/galaxy separation required modelling point sources by fitting the profiles of bright, isolated and non-saturated stars to a moffat function $(\beta=1.5)$. We used the PSF task for that purpose. This PSF model was then fitted to all objects found by DAOFIND with the ALLSTAR task.

The output parameters given by ALLSTAR (fit quality $\chi$, magnitude uncertainty $\delta m$, and sharpness $s$ ) are useful to separate a purely stellar sample from the remaining sources detected. An effective use of these parameters was made by finding the linear combination of $\chi, \delta m$ and $s$ that best separated a sample of eye-ball selected stars from a sample of eye-ball non-stellar sources (either galaxies or spurious detections around bright saturated stars and CCD defects). These eye-ball samples were selected on each chip of each image and the best linear combination was found by varying the linear coefficients and cut-off value until they led to a complete (or nearly) separation between the two samples in parameter space. This star/galaxy classifier was then applied to all objects in the list of candidates and yielded samples of stars. The final stellar samples selected were inspected on the images in order to check if star/galaxy separation was effective and that obvious stars were not wrongly discarded. The whole procedure was repeated if necessary until a satisfactory star sample selection was achieved.

This sample selection procedure was applied to both cluster (CEN and HALF fields) and background images.

\subsection{Photometry}

Due to the undersampling of point source light distributions with the WFPC2, aperture photometry tends to yield more accurate magnitude and color measurements than psf fitting. Therefore, aperture photometry was carried out on the stars selected in each image, on both the F814W and F555W images. A 2 pixel aperture radius was 
used for all WFPC2 images and for both filters, corresponding to $r=0.2^{\prime \prime}$ for the 3 Wide Field Camera (WFC) chips and $r=0.09^{\prime \prime}$ for the Planetary Camera (PC); this is a compromise between the need to include the core of the psf but avoid light contamination by neighbouring objects.

Significant but well determined aperture corrections were then applied to the measured magnitudes (Holtzman et al. 1995a). These corrections are larger for the PC chip than for the $3 \mathrm{WFC}$ ones, being about the same on the F814W and F555W filters. All the data have been zeropointed, CTE and aperture corrected following the prescriptions of Holtzman et al. (1995a,b) and Whitmore et al. (1999). The CTE corrections from the latter were used only for the shallow images in the CEN fields. For the deep exposures, the difference between the Holtzman et al. ramp correction and the Whitmore et al. timedependent one is very small.

A single reddening correction was adopted for the clusters CEN and HALF fields and for their corresponding background field. The $E(B-V)$ adopted values are listed in Table 1.

One advantage of having the partially overlapping CEN and HALF images is the ability to estimate the accuracy of our aperture photometry and to search for any systematic error in the measured magnitudes and colors. The stars in the two fields were matched by their equatorial coordinates, as provided by the astrometric solution available in the image headers. These solutions are internally consistent but often have small ( $\simeq 3-5$ pixels) offsets from one image to another. These offsets were measured by eye by superposing the estimated positions of the CEN and HALF stars. Only stars in the HALF and CEN fields with corrected positions within 1 WFC pixel of each other were considered successful matches.

The photometric comparison for the stars in common between the HALF and CEN fields of NGC 1805 is shown in Fig. 1. The upper panel shows the $m_{814}$ (solid squares) and $m_{555}$ (open circles) values. Except for the effects of saturation at the bright end in the magnitude comparison, the data lie very close to the identity lines in both cases, indicating the lack of any significant systematics in the photometry. A slight systematic effect is seen in F814W as shown by the linear fit to the data shown. This best fit solution,

$m_{\mathrm{CEN}}=0.98 m_{\mathrm{HALF}}+0.40$,

was used to bring the magnitudes of the HALF field to the same system as the CEN ones.

The few outliers in Fig. 1 were investigated further and proved to be positional $C E N$ vs. $H A L F$ mismatches that escaped our positional matching algorithm. Figure 1 is typical of all the other clusters.

\subsection{Completeness corrections}

The non-detection of faint stars by the automatic detection and star selection algorithms was quantified by

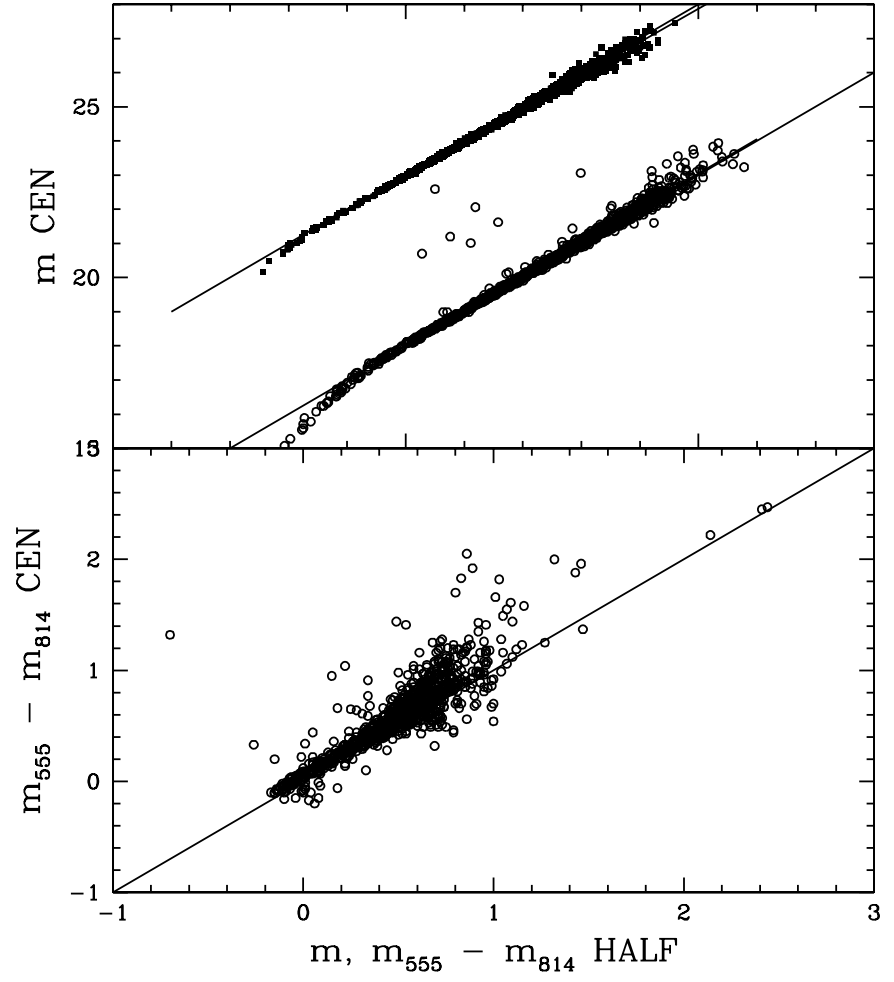

Fig. 1. Photometric consistency check for NGC 1805. $m_{814}$ and $m_{555}$ magnitudes measured in both CEN and HALF fields are compared on the upper panel. The two close straight lines in each case represent the identity function and a linear regression fit. The lower panel shows the comparison between CEN and HALF $m_{555}-m_{814}$ colours, again with the identity line for guidance

simulations of artificial stars. For each field, pointing and chip, a total of 1000 artificial stars of a fixed magnitude were added to the $\mathrm{F} 814 \mathrm{~W}$ images. These 1000 stars were placed in random positions on the image, in 10 realizations with 100 stars each. Magnitude bins of 0.5 mag width in the range $20 \leq m_{\mathrm{F} 814} \leq 26$ were covered by the experiments. Since the completeness correction depends on crowding, completeness was computed as a function of position on each CCD chip; the fraction of artificial stars recovered in each one of 64 sectors of $100 \times 100$ pixels in each chip was taken as the completeness value $c$ for that sector. To correct for incompleteness, each star was then given a weight $w$ corresponding to the distance-weighted average of $1 / c$ values of the 4 closest sectors to it:

$w_{i}=\frac{1}{\sum_{j} r_{j}^{-1}} \sum_{j} \frac{r_{j}^{-1}}{c\left(m_{i}, f, k, s_{j}\right)}$

where $i$ is an index for the star (with apparent magnitude $\left.m_{i}\right), c$ is the completeness value at its field $f$, chip $k$ and at sector $s_{j}$, whose centre lies at a distance $r_{j}$ from the star position on the chip, $j$ being an index for the 4 sectors which are closest to the star.

Figure 2 shows an example case of completeness values for the PC1 chip of the NGC 1868 CEN field. The objects detected in the chip are shown as dots in the figure as a 


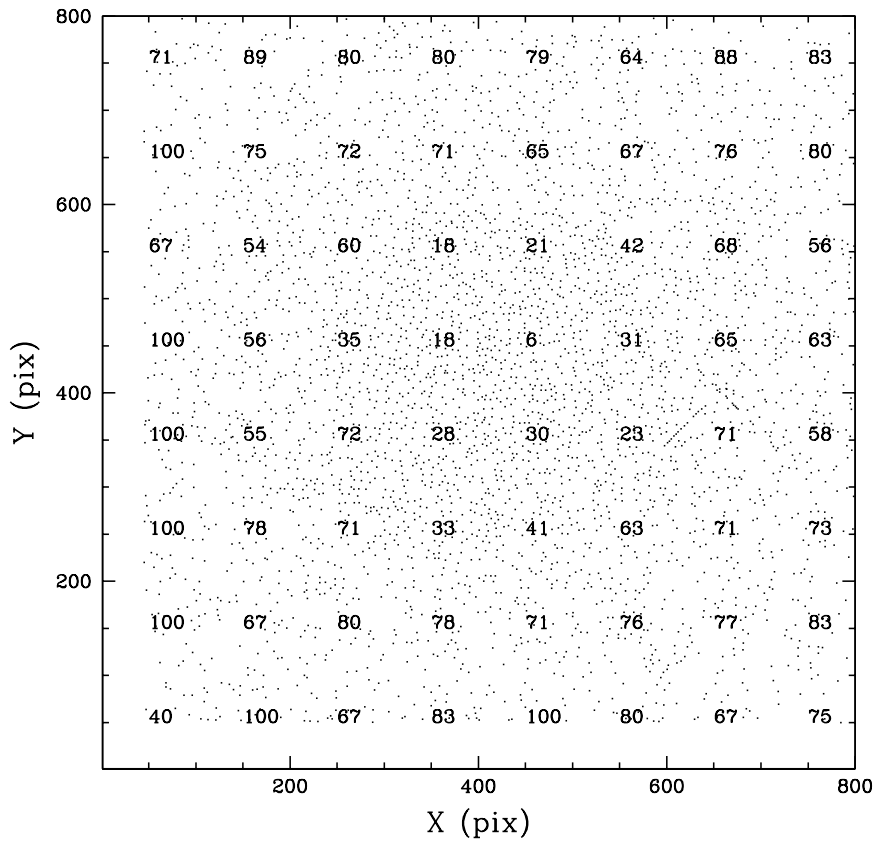

Fig. 2. Completeness values for NGC 1868 CEN field, chip 1 (PC), $23<m_{814}<23.5$, calculated as described in Sect. 2.3. The completeness map is shown as percentage values superposed to the distribution of detected objects on the chip

function of $X$ and $Y$ positions. The completeness values for $23.0 \leq m_{814} \leq 23.5$ found by the procedure described above are also shown for each sector on the chip. There is a clear anticorrelation between crowding and completeness, as expected: in the central cluster regions completeness is significantly smaller than in the outer areas.

Completeness functions were also computed for the parallel WFPC2 fields used for field star subtraction. The low stellar density of these fields allowed us to use a single completeness value for each magnitude bin.

\section{Density profiles and luminosity functions}

In order to build density or surface brightness profiles and to search for variations in the shape of the luminosity function with position within each cluster, the stars found in the HALF and CEN fields had to be put together into a single sample. This combined sample included all the stars found in the CEN field plus the stars in the HALF field located outside the CEN field borders. We again used the astrometric information available in the image headers (plus any additional offset correction; see Sect. 2.2) to find the equatorial coordinates (2000 EPOCH) corresponding to the CEN field chip borders.

Figure 3 shows the distribution on the sky of the combined sample of stars for NGC 1805. The extra stars added by the HALF field are shown with different symbols for clarity.

An important factor to be accounted for when computing the profiles and luminosity functions for each cluster is the increasing fractional loss of sampled area in the sky as a function of angular distance from the cluster's

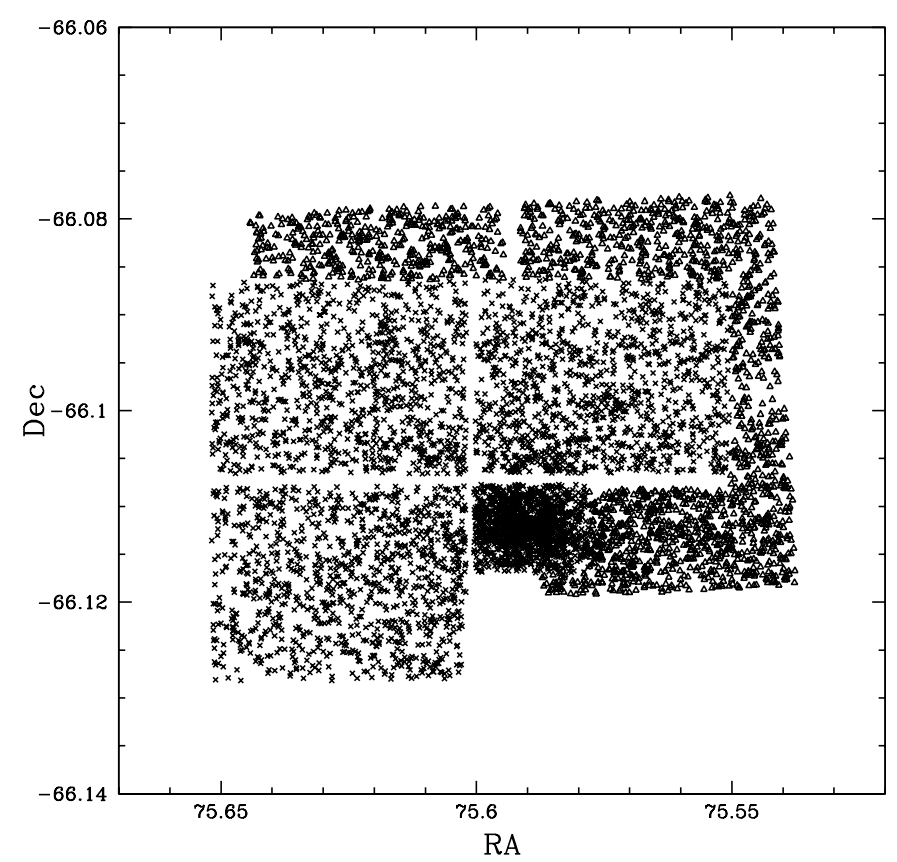

Fig. 3. Distribution on the plane of the sky of stars in NGC 1805. The crosses (triangles) represent stars in the CEN (HALF) field, the cluster centre coinciding with the CEN field PC chip

center. The profiles and luminosity functions were considered only out to radii where the area sampled by the combined CEN and HALF fields was larger than $30 \%$ of the total for that radius. This typically corresponded to $R \lesssim 25$ pc (roughly 1000 pixels). Within these limits, each star in our sample was given an additional weight corresponding to the inverse of the fractional sampled area at its distance from the cluster centre. This fraction was computed by randomly throwing points within each ring of a fixed radius and determining the percentage of such points within either the CEN or HALF field borders. Therefore, the final weight assigned to each star was:

$W_{i}=\frac{w_{i}}{f\left(r_{i}\right)}$,

where $i$ is an index for the stars, $w_{i}$ is given by Eq. (1), and $f\left(r_{i}\right)$ is simply

$\frac{A_{\text {samp }}\left(r_{i}\right)}{A_{\text {tot }}\left(r_{i}\right)}$,

where $A_{\text {samp }}\left(r_{i}\right)$ is the sampled area (CEN or HALF fields added together) and $A_{\text {tot }}=2 \pi r_{i} \mathrm{~d} r_{i}$ is the total area of a ring of width $\mathrm{d} r_{i}$ at a distance $r_{i}$ from the cluster centre.

In the next subsections we show density and surface brightness profiles and luminosity functions for each cluster in the sample.

\subsection{NGC 1805}

Figure 4 shows the number density and surface brightness profiles of NGC 1805 out to the largest possible radii available. The profiles were built by adding up the weighted 


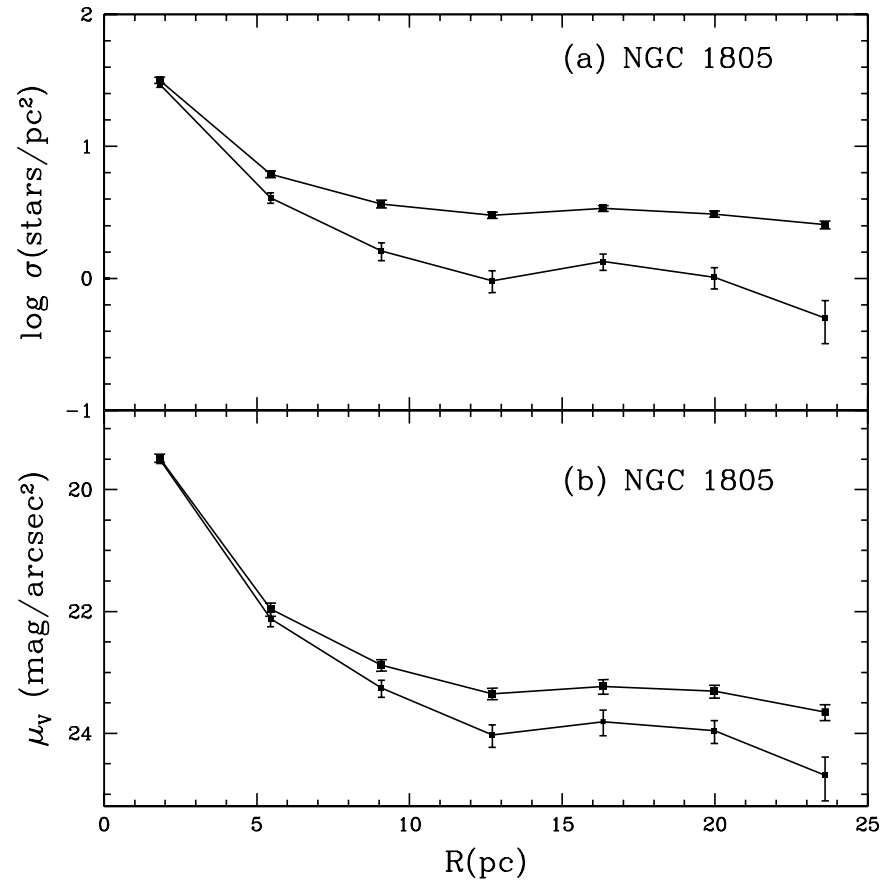

Fig. 4. Stellar surface density (panel a)) and surface brightness (panel b)) profiles for NGC 1805. The upper curves are not corrected for background star contamination. The corrected profiles are the lower curves

number of stars in rings 150 pixels $(\simeq 3.6 \mathrm{pc})$ wide centered on the cluster. Each star contributed to the profile with a weight given by Eqs. (1) and (2) as explained in the previous section.

The upper curves in Fig. 4 are not corrected for field star contamination. They roughly flatten at $R \simeq 10 \mathrm{pc} \simeq$ 410 pixels from the cluster's center. The lower curves show the cluster profiles after the contribution of field stars in the background was subtracted off.

From its light profile we were able to estimate the halflight radius of NGC 1805 to be $R_{\mathrm{hl}} \simeq 1.8 \mathrm{pc}$.

In Fig. 5 we show the distribution of stars as a function of absolute magnitude $M_{555}\left(\Phi\left(M_{555}\right)\right.$ or luminosity function) for different regions of the cluster. The $M_{555}$ values assume a distance modulus of $m-M=18.5$ for the LMC (Panagia et al. 1991). Only main-sequence stars were considered and the field star contamination was subtracted off using the background WFPC2 field. Even though luminosity functions could extend brightwards in the CEN field, we did not analyze the luminosity function bright end because saturation sets in at $M_{555} \simeq 18.5-19$ for stars in the HALF field, therefore considerably reducing the available solid angle. All the luminosity functions were scaled to the total cluster area used to build the global luminosity function, shown in panel $a$ of each figure.

Panel 5a shows the global luminosity function within $R=1050$ pix $(\simeq 25 \mathrm{pc})$. The other panels probe different distances from the centre, as indicated. The luminosity function in the innermost radius is clearly flattened compared to the ones further out, there being in fact a systematic trend of increase in the luminosity function slopes

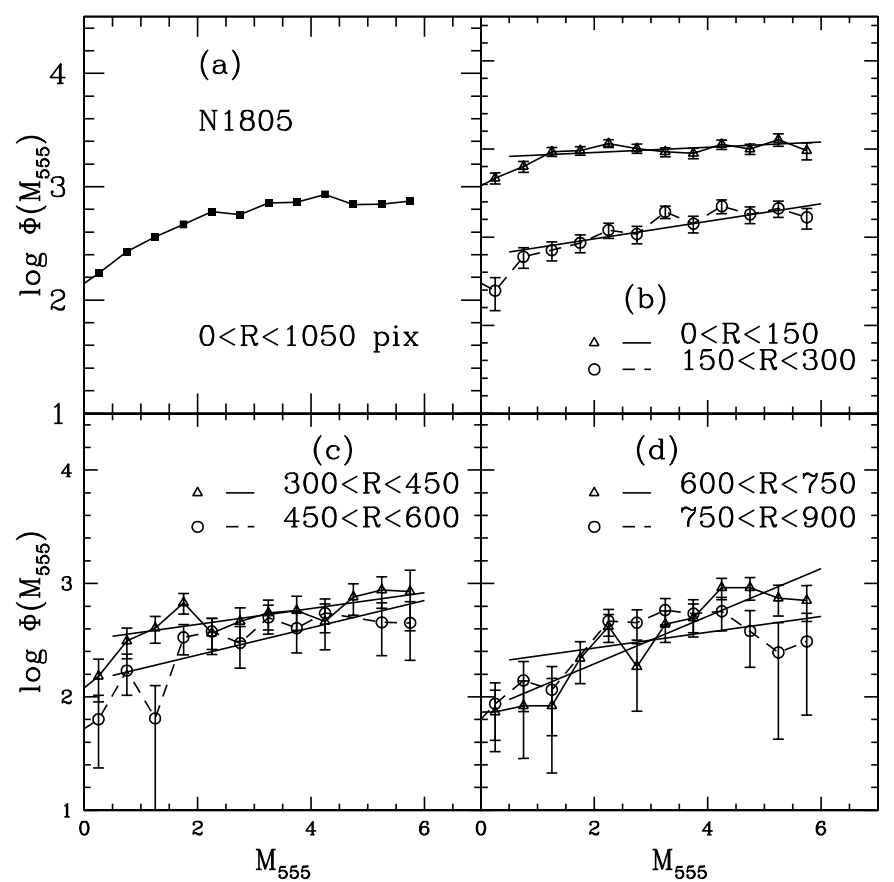

Fig. 5. Positional dependent luminosity functions for NGC 1805. The range in radius to which each luminosity function corresponds is indicated in each panel (1 pc = 41 pix). The luminosity functions have been corrected for background contamination as explained in the text. Linear fits to the luminosity functions are shown on each panel. The best fit lines bracket the range in $M_{555}$ used in the fit

with distance from the cluster centre. Linear fits were carried out for each luminosity function and are also shown in Fig. 5. With the exception of the last radial ring, a powerlaw in luminosity is a good description of the luminosity function shapes.

The best-fit slope values are plotted in Fig. 6. The systematic increase in the slopes is clear out to about $15 \mathrm{pc}$ from the center. There is a drop in the luminosity function slope in the last radial ring, but the density contrast above the background is small and therefore the statistics are noisier. Given the young age of this cluster (Johnson et al. 2000), the observed mass segregation in NGC 1805 possibly reflects the initial conditions during cluster formation (see Sect. 4).

\subsection{NGC 1818}

Figure 7 shows the stellar density and surface brightness profiles of NGC 1818. The symbols are the same as in Fig. 4. NGC 1818 is more extended $\left(R_{\mathrm{hl}}=2.6 \mathrm{pc}\right)$ and has a steeper profile than NGC 1805 in the outer regions; its corrected profiles systematically decline out to the outermost radius shown. At $r \simeq 18 \mathrm{pc}$ the cluster density has fallen by about a factor of 50 from its central value of $\sigma(0) \simeq 40$ stars $/ \mathrm{pc}^{2}$.

Luminosity functions for various rings (again each 3.6 pc wide) centred on NGC 1818 are shown in Fig. 8. As in NGC 1805, the luminosity function slopes tend to 


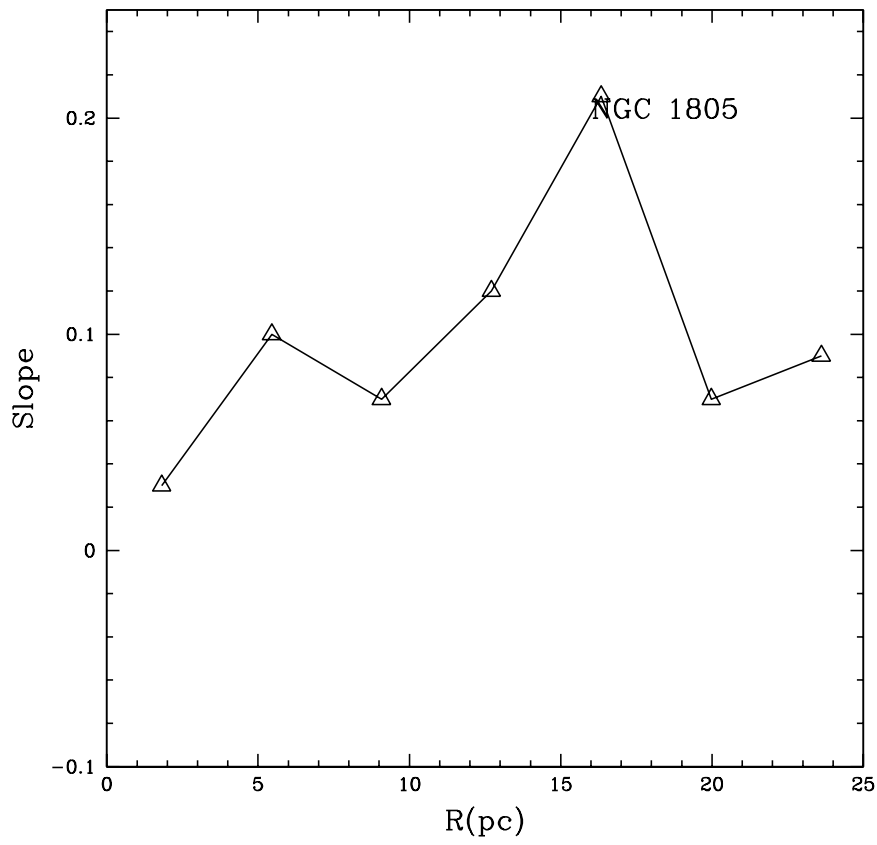

Fig. 6. Slope values obtained from linear fits to the luminosity functions shown in Fig. 5 shown as a function of distance from cluster's centre

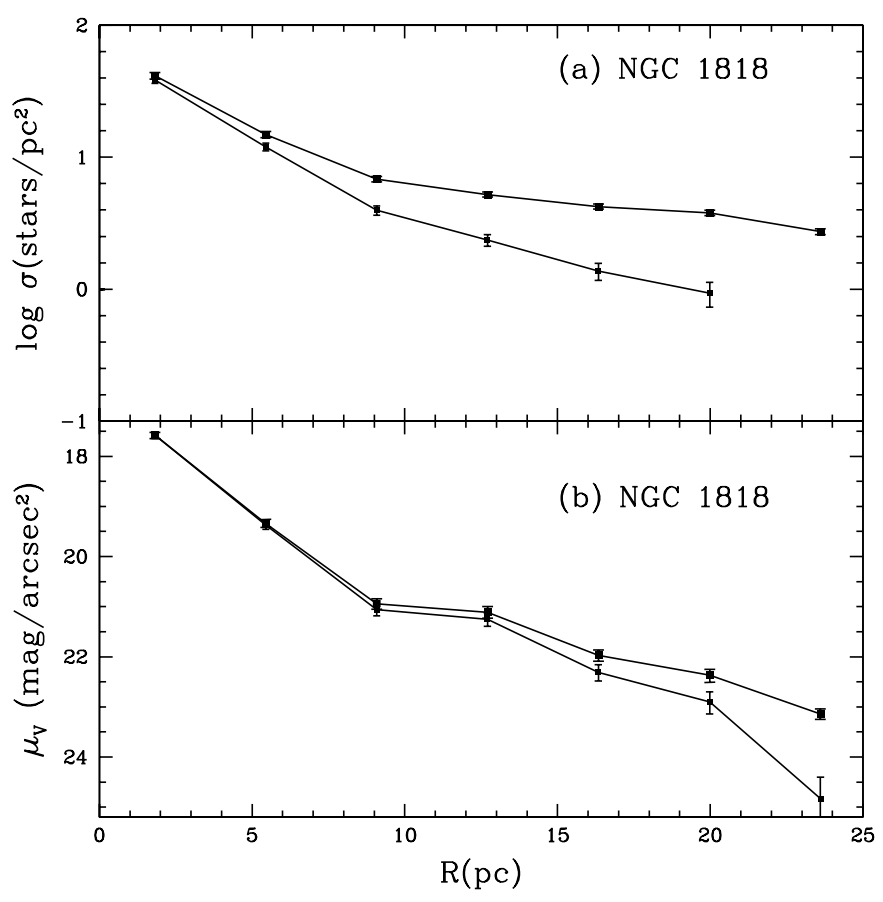

Fig. 7. Stellar surface density (panel a)) and surface brightness (panel b)) profiles for NGC 1818. The upper curves are not corrected for background star contamination. The corrected profiles are the lower curves

systematically increase with radius out to $R \simeq 17 \mathrm{pc}$ from the centre (Fig. 9). A drop in the luminosity function slope is seen in the outermost radius of both clusters. The observed positional dependence of the luminosity function slopes may again be primordial, since NGC 1818 is also a young system (Johnson et al. 2000).

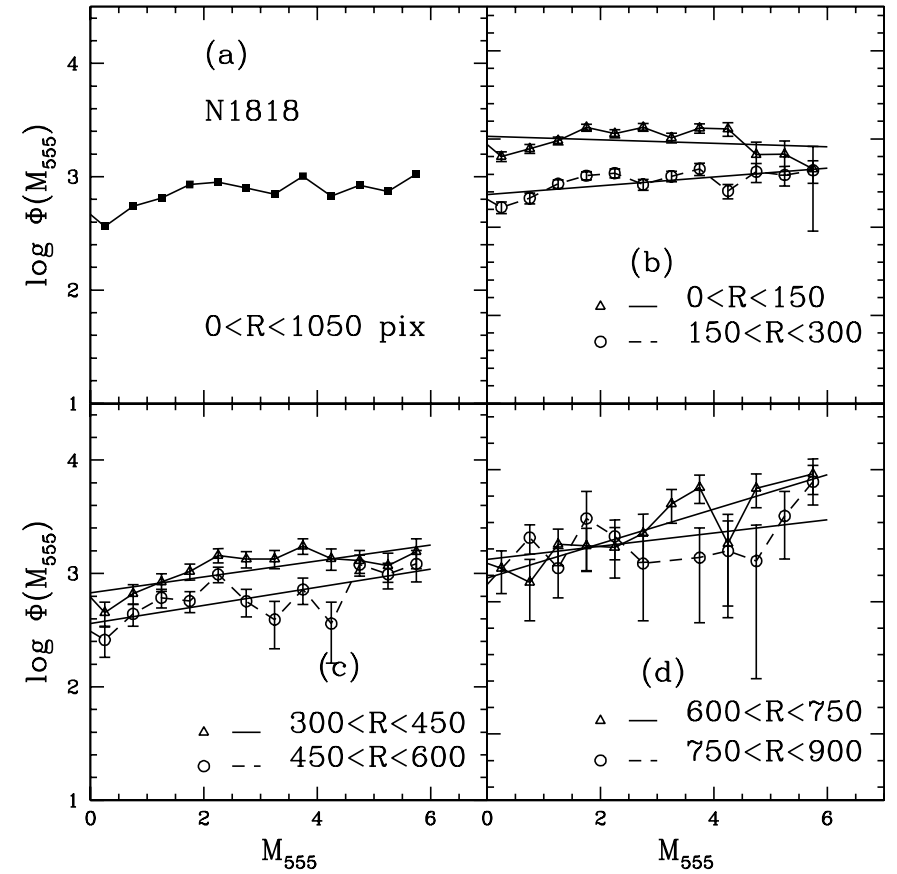

Fig. 8. Positional dependent luminosity functions for NGC 1818. The range in radius to which each luminosity function corresponds is indicated in each panel (1 pc = 41 pix). The luminosity functions have been corrected for background contamination as explained in the text. Linear fits to the luminosity functions are shown on each panel. The best fit lines bracket the range in $M_{555}$ used in the fit

\subsection{NGC 1831}

The density and surface brightness profiles of NGC 1831 are shown in Fig. 10. The number density profile is steeper than that of NGC 1818 but the surface brightness profile is shallower. NGC 1831 is richer and has a higher central density of stars $\left(\sigma \simeq 50 \mathrm{stars} / \mathrm{pc}^{2}\right)$ than either NGC 1805 or NGC 1818, but its central surface brightness is lower than that of NGC 1818. We obtain a half-light radius of $R_{\mathrm{hl}} \simeq 3.6 \mathrm{pc}$ from the light distribution of NGC 1831 .

The positional dependent luminosity functions are shown in Fig. 11. Comparison of the luminosity functions in panel 11b with those in panel 11c shows that the inner 7 or 8 pc ( $R \lesssim 300$ pix $)$ contains a larger relative proportion of luminous $\left(M_{V} \lesssim 2\right)$ stars. Notice that the luminosity function in the innermost radial bin shows an abrupt drop at $M_{555}>5.5$. This could be an artificiality caused by an overestimated completeness function. A less abrupt drop is present in the central luminosity function of NGC 1818 as well. A straight line in $\log \Phi$ vs. $M_{555}$ seems to be a better description of the luminosity function shapes in NGC 1831 than it was for NGC 1818.

Mass segregation is also confirmed in Fig. 12, where the best-fit slopes to the luminosity functions are plotted; the luminosity function slopes of the 2 inner radii are smaller than those in the other rings, where the slopes roughly flatten out. 


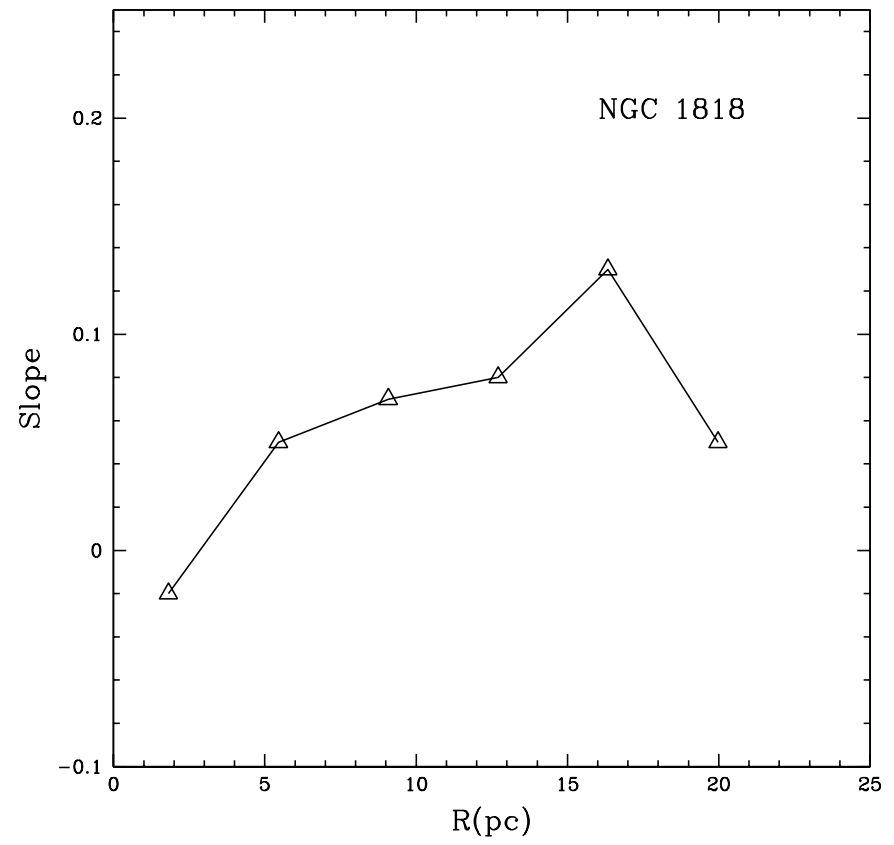

Fig. 9. Slope values obtained from linear fits to the luminosity functions shown in Fig. 8 shown as a function of distance from cluster's centre

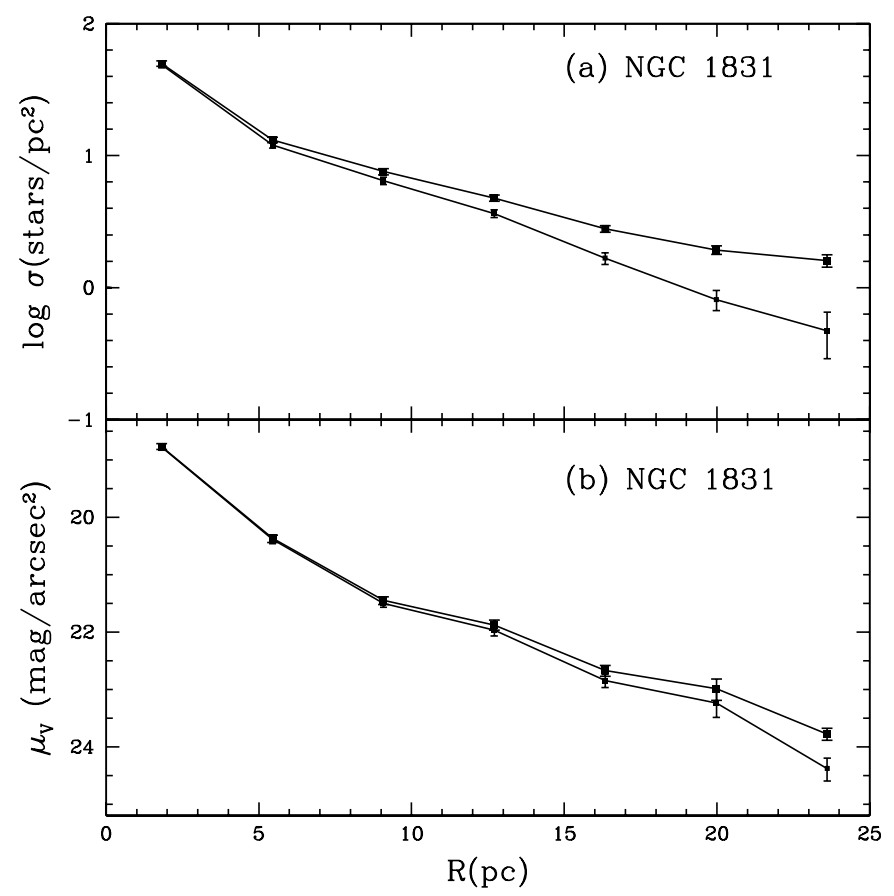

Fig. 10. Stellar surface density (panel a)) and surface brightness (panel b)) profiles for NGC 1831. The upper curves are not corrected for background star contamination. The corrected profiles are the lower curves

\subsection{NGC 1868}

This is a richer cluster than the previous ones, with a central stellar density of about 60 stars $/ \mathrm{pc}^{2}$. NGC 1868 density and surface brightness profiles extend out nearly to the largest radius used, as shown by Fig. 13. It has a steeper profile than NGC 1831, especially in the inner

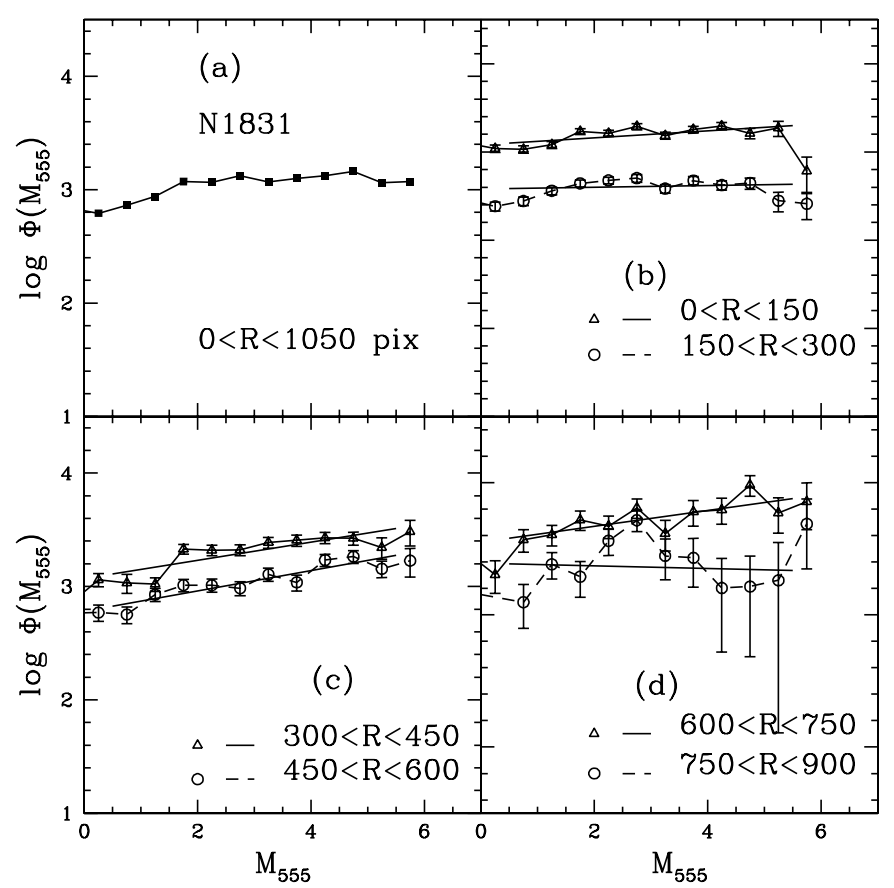

Fig. 11. Positional dependent luminosity functions for NGC 1831. The range in radius to which each luminosity function corresponds is indicated in each panel ( $1 \mathrm{pc}=41 \mathrm{pix})$. The luminosity functions have been corrected for background contamination as explained in the text. Linear fits to the luminosity functions are shown on each panel. The best fit lines bracket the range in $M_{555}$ used in the fit

regions. In fact, the profiles shown are the steepest of all clusters in our sample. From its light profile we estimate a half-light radius of only about $R_{\mathrm{hl}} \simeq 1.8 \mathrm{pc}$.

Figures 14 and 15 show how the luminosity function varies as a function of position within the cluster. The luminosity function fit in the innermost radial bin is strongly affected by a drop in the luminosity function at the faintest magnitude bin, which could again indicate overestimated completeness at this magnitude level. But the effect of mass segregation in NGC 1868 is still clearly visible, with steeper luminosity functions in the outer regions relative to the inner ones. The systematic in the slope is clear in Fig. 15 as well, where the slope continually rises out to $R \simeq 10$ pc, roughly flatenning out beyond this point.

\subsection{NGC 2209}

NGC 2209 is a considerably sparser cluster than the previous ones. It has the lowest central density in both stars and light $\left(\sigma(0) \simeq 11 \mathrm{stars} / \mathrm{pc}^{2} ; \mu_{V} \simeq 21.6 \mathrm{mag} / \mathrm{arcsec}^{2}\right)$, as attested by Fig. 16. Its half-light radius is large: $R_{\mathrm{hl}} \sim$ $7.6 \mathrm{pc}$, its light profile is therefore the shallowest in our sample.

Figure 17 shows that a power-law in luminosity is a poor description for the luminosity function in NGC 2209. But the linear fits and derived slopes, despite not always reflecting the complexity in the luminosity function shapes, can be still useful as guidelines and help quantify 


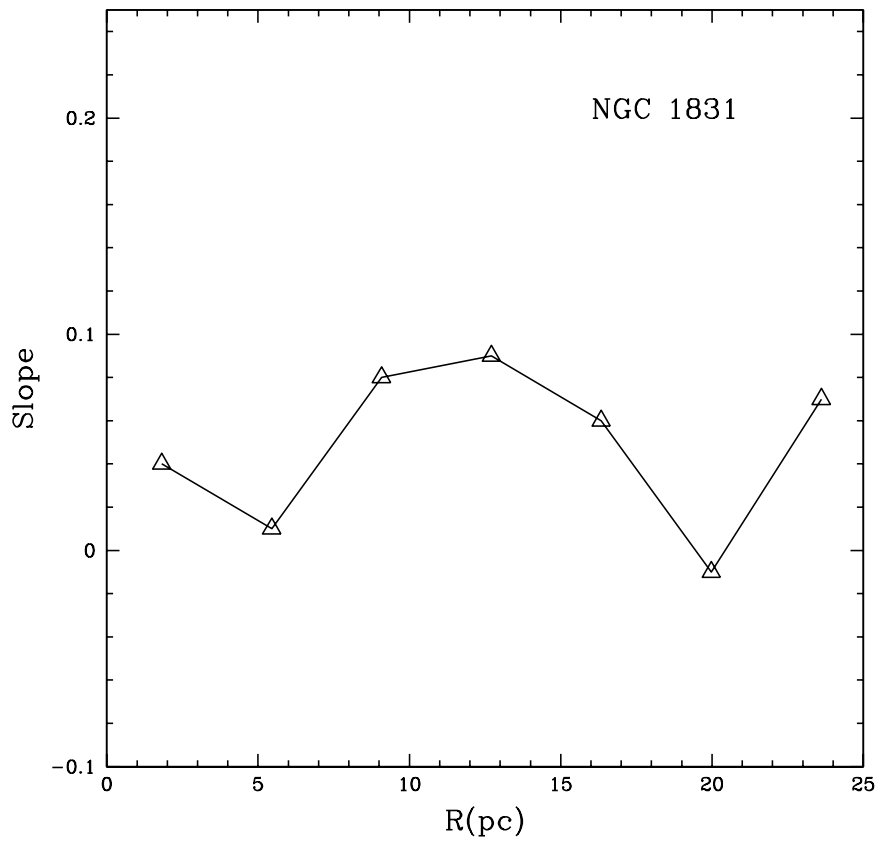

Fig. 12. Slope values obtained from linear fits to the luminosity functions shown in Fig. 11 shown as a function of distance from cluster's centre

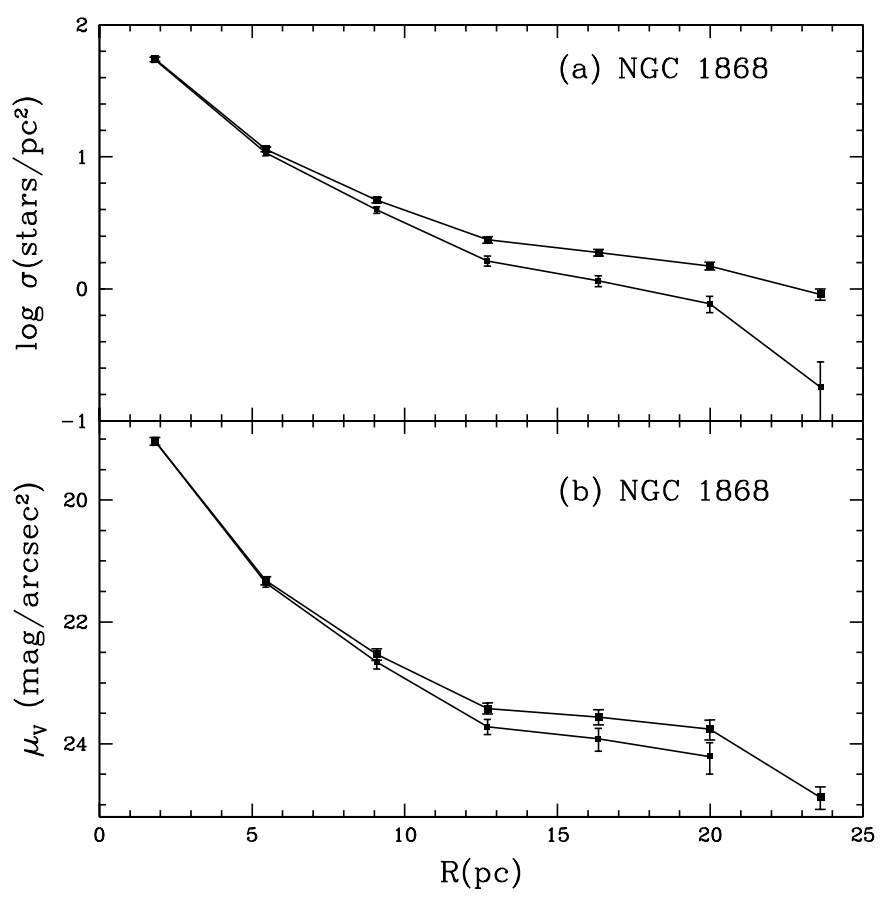

Fig. 13. tellar surface density (panel a)) and surface brightness (panel b)) profiles for NGC 1868. The upper curves are not corrected for background star contamination. The corrected profiles are the lower curves

the amount of mass segregation. Just as in the previous cases, the drop in the luminosity function at the faintest magnitudes, especially in the central region, could signal a problem with completeness corrections. The slope values derived from fits to the luminosity functions in Fig. 17 are shown in Fig. 18. Considered individually, they are somewhat dependent on the adopted fit interval, but the

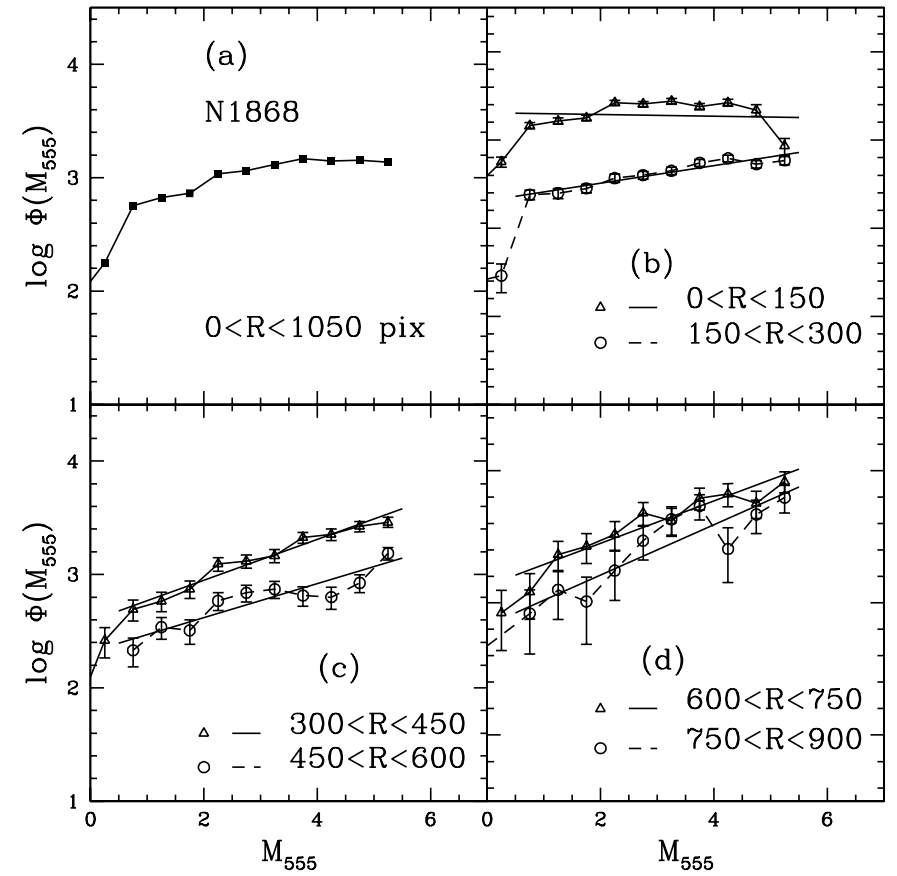

Fig. 14. Positional dependent luminosity functions for NGC 1868. The range in radius to which each luminosity function corresponds is indicated in each panel ( $1 \mathrm{pc}=41 \mathrm{pix})$. The luminosity functions have been corrected for background contamination as explained in the text. Linear fits to the luminosity functions are shown on each panel. The best fit lines bracket the range in $M_{555}$ used in the fit

steepening in the luminosity functions is continuous and in the case of NGC 2209 extends out to the largest radius studied.

\subsection{Hodge 14}

Similarly to NGC 2209, Hodge 14 is also a low density and low-surface brightness cluster $\left(\sigma(0) \simeq 12\right.$ stars $/ \mathrm{pc}^{2}$; $\left.\mu_{V} \simeq 21 \mathrm{mag} / \operatorname{arcsec}^{2}\right)$. The profiles are shown in Fig. 19; wider bins in radius $(\Delta R=200$ pixels $=\simeq 5 \mathrm{pc})$ were used to reduce noise. Contrary to all other clusters, there is little or no excess of stars beyond 15 pc away from the centre of Hodge 14.

H14's luminosity functions are noisier than the previous ones. As in NGC 2209, a power-law in luminosities is a poor description of most luminosity function shapes seen in Fig. 20. Yet, with the wider binning used, it is possible to see that its luminosity function slopes display a similar behaviour to the other clusters: a continuous rise out to some radius (in H14's case, $R \simeq 10-12 \mathrm{pc}$ ) and a drop or flattening beyond that point (Figs. 20 and 21).

The structural parameters derived from the profiles of all 6 clusters discussed above are listed in Table 2 .

\section{Discussion}

Our first concern has been to check for any systematic effects in our derived profiles and luminosity functions. 


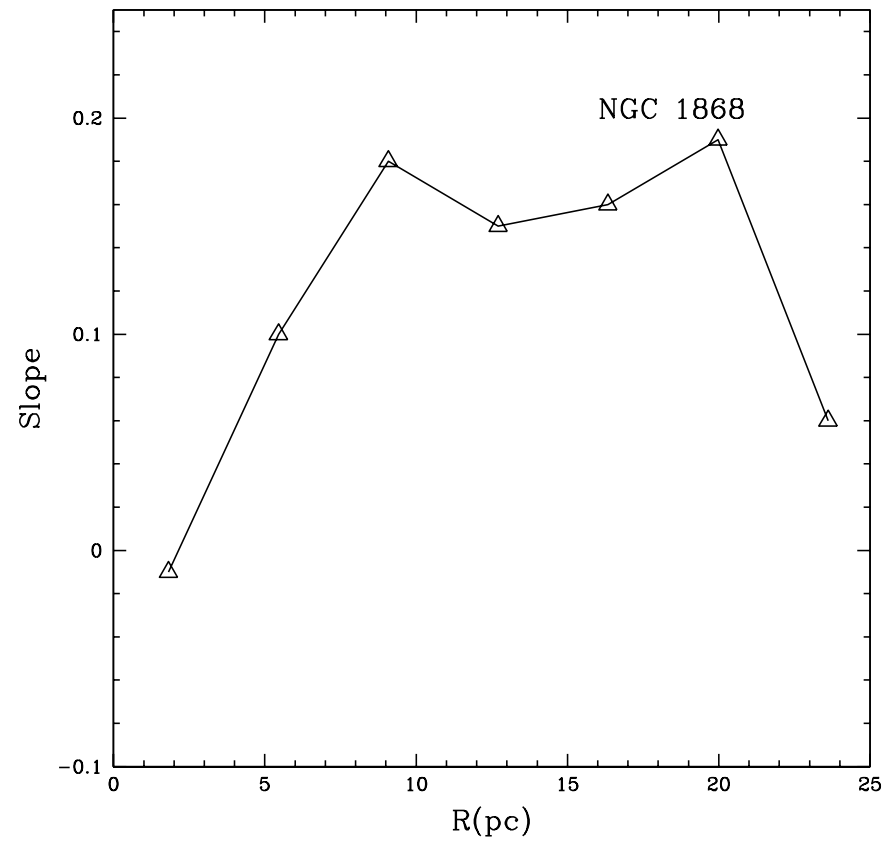

Fig. 15. Slope values obtained from linear fits to the luminosity functions shown in Fig. 14 shown as a function of distance from cluster's centre

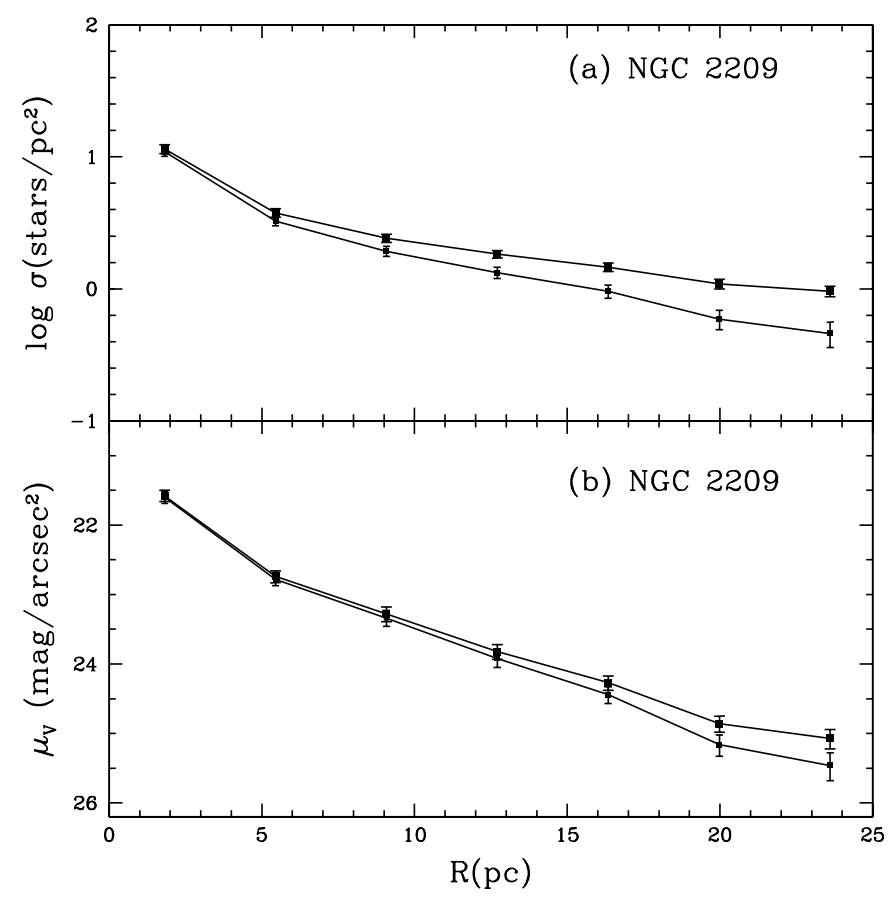

Fig. 16. Stellar surface density (panel a)) and surface brightness (panel b)) profiles for NGC 2209. The upper curves are not corrected for background star contamination. The corrected profiles are the lower curves

There is plenty of photometric work on several of our clusters, but mostly from ground based observations and using different photometric systems (Vallenari et al. 1993; Ferraro et al. 1995; Geisler et al. 1997). In Fig. 22 we compare the surface brightness profiles obtained by Elson (1991) for four of our clusters with the ones shown in the previous section. Both sets of profiles display a general

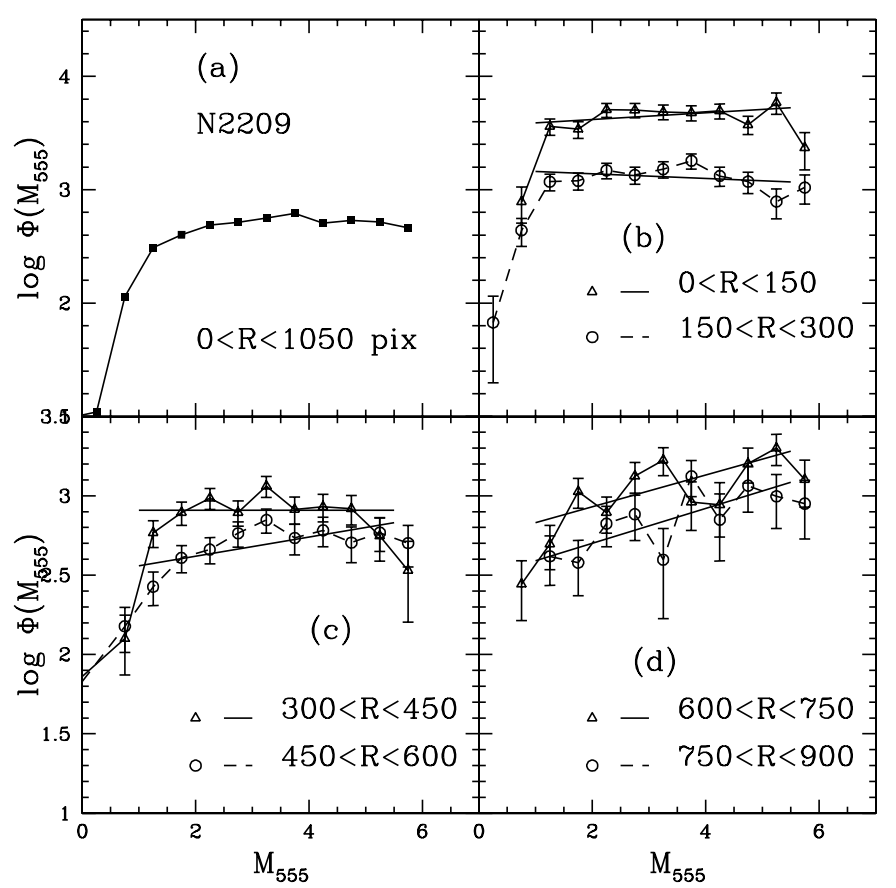

Fig. 17. Positional dependent luminosity functions for NGC 2209. The range in radius to which each luminosity function corresponds is indicated in each panel ( $1 \mathrm{pc}=41 \mathrm{pix})$. The luminosity functions have been corrected for background contamination as explained in the text. Linear fits to the luminosity functions are shown on each panel. The best fit lines bracket the range in $M_{555}$ used in the fit

Table 2. Cluster's structural parameters from density and surface brightness profiles

\begin{tabular}{|c|c|c|c|}
\hline Cluster Name & $\mathrm{e} \sigma_{0}\left(\operatorname{stars} / p c^{2}\right) \mu_{V}(0)$ & $\left(\mathrm{mag} / \operatorname{arcsec}^{2}\right)$ & $R_{\mathrm{hl}}(\mathrm{pc})$ \\
\hline NGC 1805 & 30 & 19.5 & 1.8 \\
\hline NGC 1818 & 36 & 17.9 & 2.6 \\
\hline NGC 1831 & 49 & 18.8 & 3.6 \\
\hline NGC 1868 & 54 & 19.0 & 1.8 \\
\hline NGC 2209 & 11 & 21.6 & 7.6 \\
\hline Hodge 14 & 12 & 21.1 & 2.9 \\
\hline
\end{tabular}

agreement. Our NGC 1868 profile seems to systematically include more light beyond $R \sim 5$ pc. There are several possible reasons for this small discrepancy such as overestimated sky subtraction in the integral photometry by Elson (1991) or an overcorrection for completeness effects on our data. But given the entirely different photometric methods which led to the profiles shown in the figure, the level of agreement is evidence that there are no strong systematic effects in the photometry, sample selection and background subtraction in either work.

There is a relative paucity of deep enough luminosity functions published in the literature for LMC clusters to enable a direct comparison to our data. Vallenari et al. (1992) have obtained $B V$ photometry for NGC 1831 down to $V \lesssim 22$ and derived a cumulative luminosity function down to $V \simeq 21$. This limit is close to the bright 


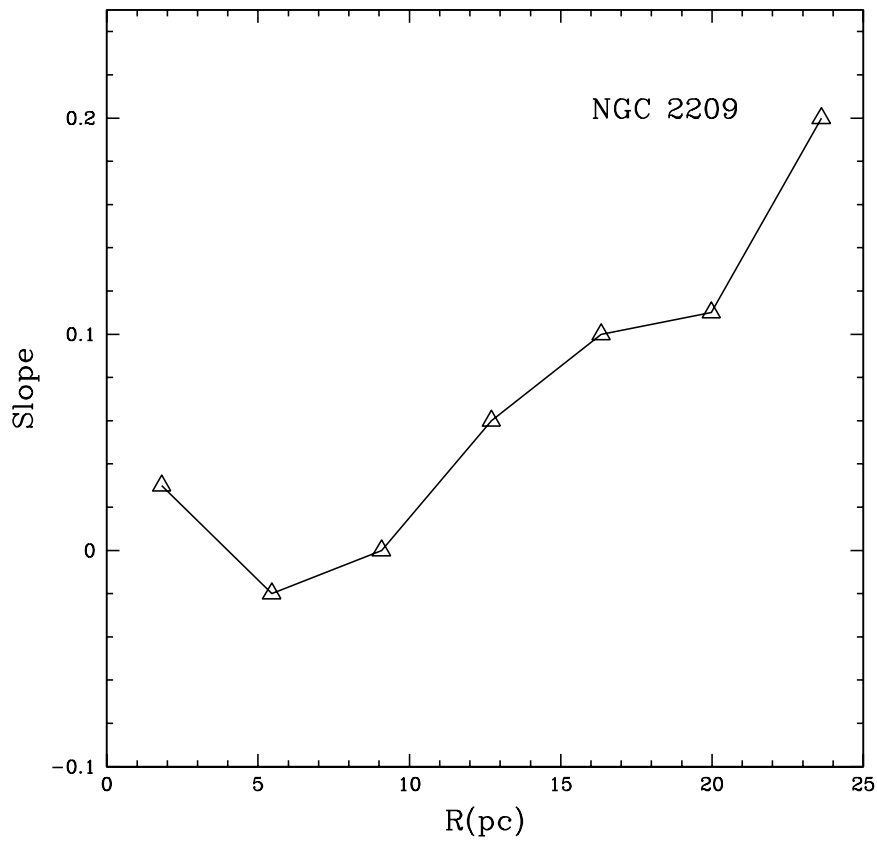

Fig. 18. Slope values obtained from linear fits to the luminosity functions shown in Fig. 17 shown as a function of distance from cluster's centre

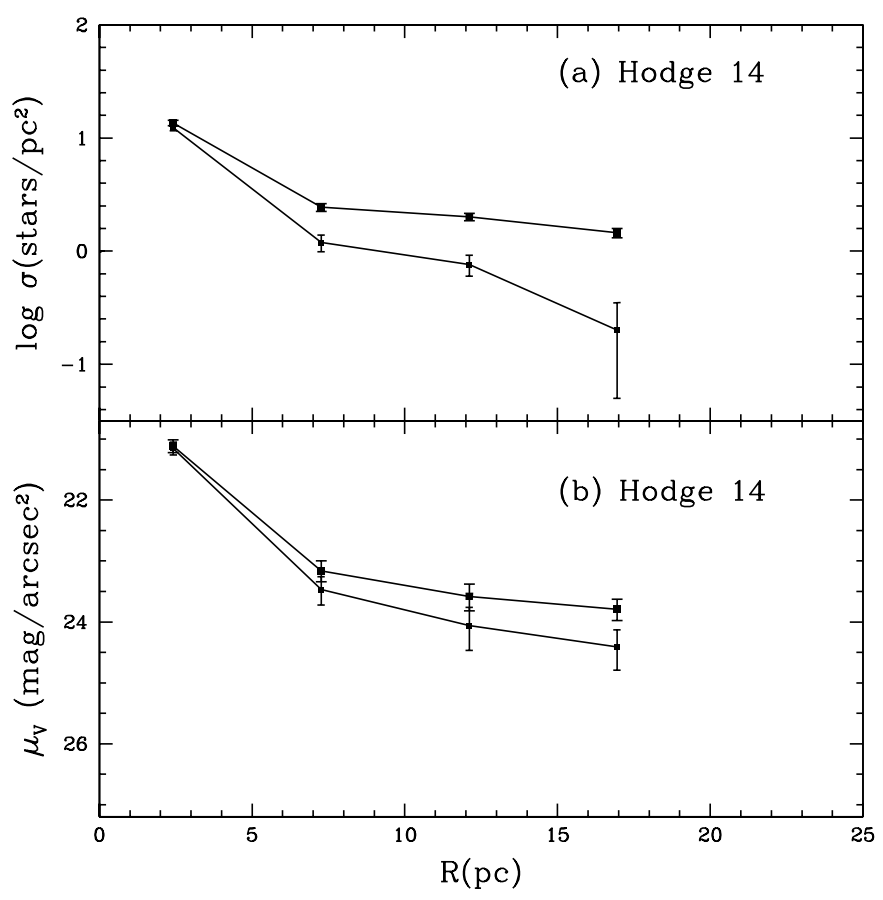

Fig. 19. Stellar surface density (panel a)) and surface brightness (panel b)) profiles for Hodge 14. The upper curves are not corrected for background star contamination. The corrected profiles are the lower curves

limit of our sample and the overlap in magnitudes is too small to allow a comparison. A similar difficulty occurs with the luminosity functions for NGC 1868 by Flower et al. (1980) and for NGC 1818 by Will et al. (1995). Olsen et al. (1998) analyzed deep HST/WFPC2 CMDs for 6 old globular clusters in the LMC, deriving their ages, metallicities and horizontal branch structures, but did not

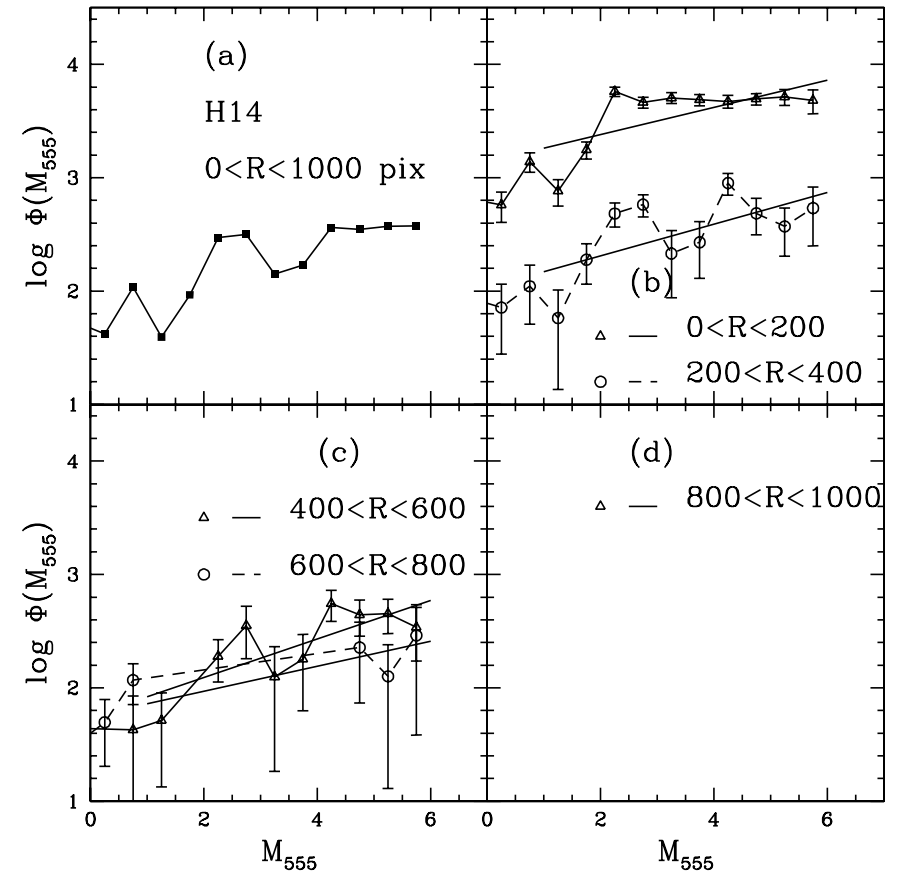

Fig. 20. Positional dependent luminosity functions for Hodge 14. The range in radius to which each luminosity function corresponds is indicated in each panel $(1 \mathrm{pc}=41 \mathrm{pix})$. The luminosity functions have been corrected for background contamination as explained in the text. Linear fits to the luminosity functions are shown on each panel. The best fit lines bracket the range in $M_{555}$ used in the fit

investigate their luminosity functions. Elson et al. (1998c) also show an HST CMD of NGC 1818 and discuss its binary sequence but do not use the data for deriving a luminosity function.

For NGC 1818, however, Hunter et al. (1997) have obtained deep HST photometry, also using the F555W and F814W filters. Figure 23 shows the comparison between their global mass function and ours. The conversion from luminosity to mass in our sample was done using a suitable isochrone from Worthey et al. (1999) in a similar way as in Santiago et al. (1996) and Santiago et al. (1998). We do not cover the high mass end of the Hunter et al. data since we are again restricting the mass range to that sampled by the deep (large exposure time) HALF field. But the agreement between the two mass functions in the common mass range is striking. We thus, conclude that the present data, besides making up a homogeneous dataset, lack any strong systematics due to sample selection or completeness.

How similar are the mass functions and their systematic trend with position among our sample clusters? Are our data consistent with a universal shape for the IMF? These questions are not easily answered without careful attention to two important issues: a) Derivation of present day mass functions (PDMFs) from luminosity functions require a mass-luminosity relation, which in turn depends on metallicity and age. Thus, this question is intimately related to finding the best fitting isochrones to the cluster CMDs and checking their sensitivity to different 


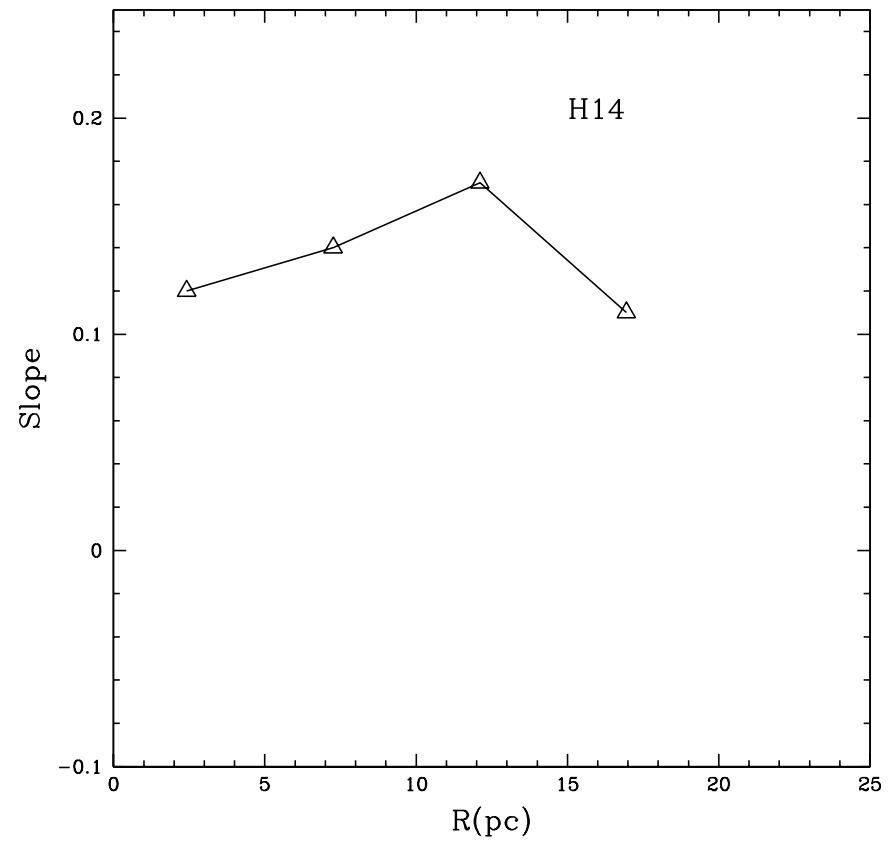

Fig. 21. Slope values obtained from linear fits to the luminosity functions shown in Fig. 20 shown as a function of distance from cluster's centre

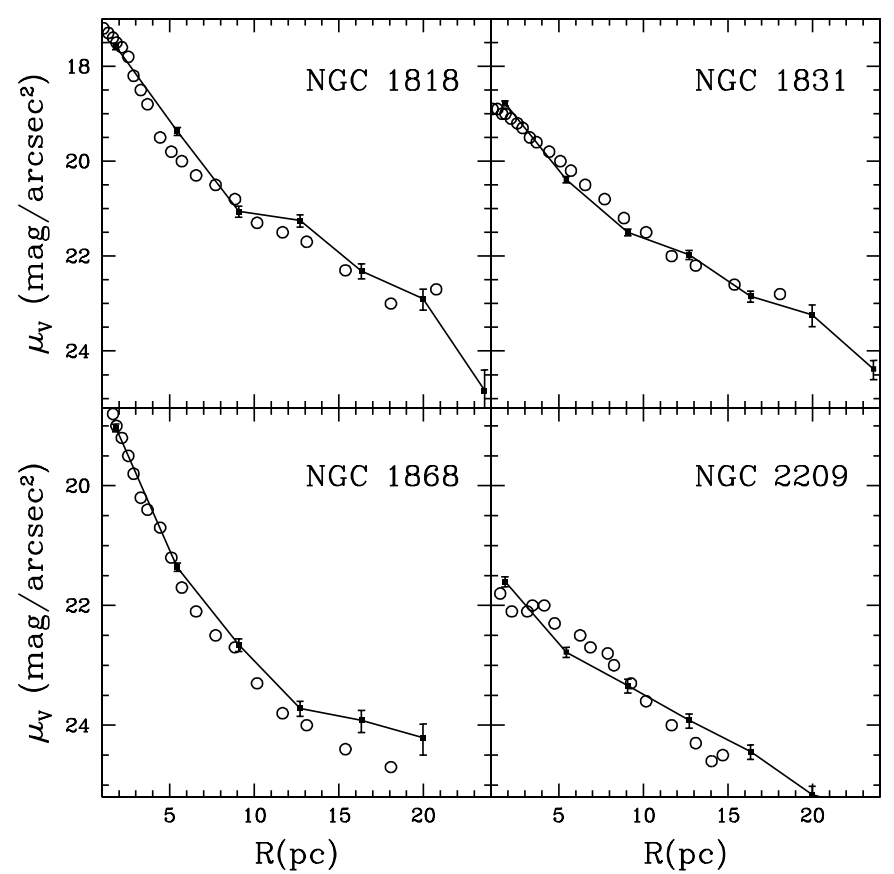

Fig. 22. Background corrected surface brightness profiles for 4 clusters in our sample compared to those from Elson (1991), based on integral photometry. The open circles are the data from Elson (1991), while the solid points are from the present paper

evolutionary models. This is currently underway for our clusters CMDs; b) Recovering the IMF from the PDMF requires reconstruction of the dynamical history of the cluster, something more easily done with the help of $N$-body simulations.

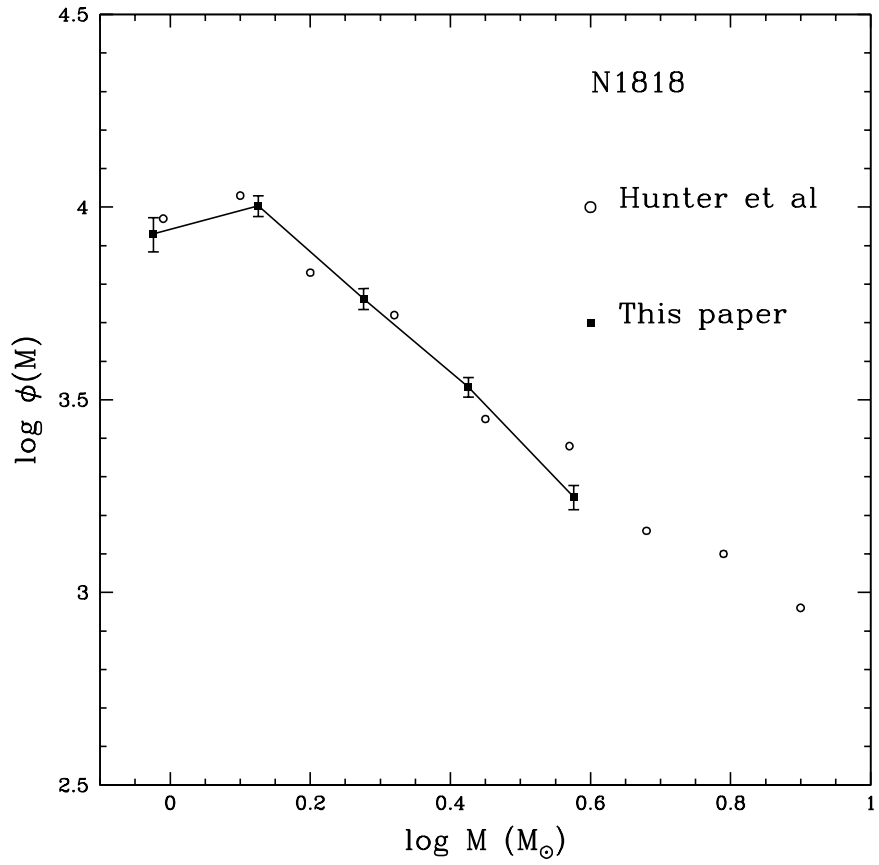

Fig. 23. Comparison of the NGC 1818 mass function from this work to that of Hunter et al. (1997). The symbols are as indicated

At present, however, we concentrate on the 2 youngest clusters in the sample, NGC 1805 and NGC 1818, whose ages are below $\tau \simeq 4010^{6}$ years. Figure 24 shows the global mass functions for both clusters (panel a), plus the mass functions in an outer region (panel b). The two mass functions differ, that of NGC 1805 being steeper. These mass functions likely reflect the IMF in both cases, especially for the outer radius shown, where the dynamical timescales for $M \lesssim 3 M_{\odot}$ stars are $\gtrsim 10^{9}$ yrs, somewhat longer than the clusters ages. For the outer region, the mass function slopes, $\Gamma=\mathrm{d} \log \phi(M) / \mathrm{d} \log (M)$, are -1.5 for NGC 1818 and -2.4 for NGC 1805, therefore steeper than the Salpeter value of $\Gamma=-1.35$. This result suggests that the shape of the the IMF may vary with environment or metallicity (see Scalo 1998, for a recent review on IMF variations).

Note however, that Elson et al. (1998c) have analyzed the binary fraction of NGC 1818 using HST $V$ vs. $U-V$ data and have found it to increase inwards. The authors argue that this is consistent with dynamical evolution. In fact, unresolved binaries may have an effect on the observed luminosity function or mass function shape. We are currently using statistical tools and modelling techniques that make use of the full two-dimensional information available from the cluster CMDs, in order to derive binary fractions and mass function shapes simultaneously for each cluster, and investigate their variation with distance from the centre.

\section{Summary}

We have used extensive WFPC2 photometry of stars in 6 rich star clusters in the LMC in order to obtain and 


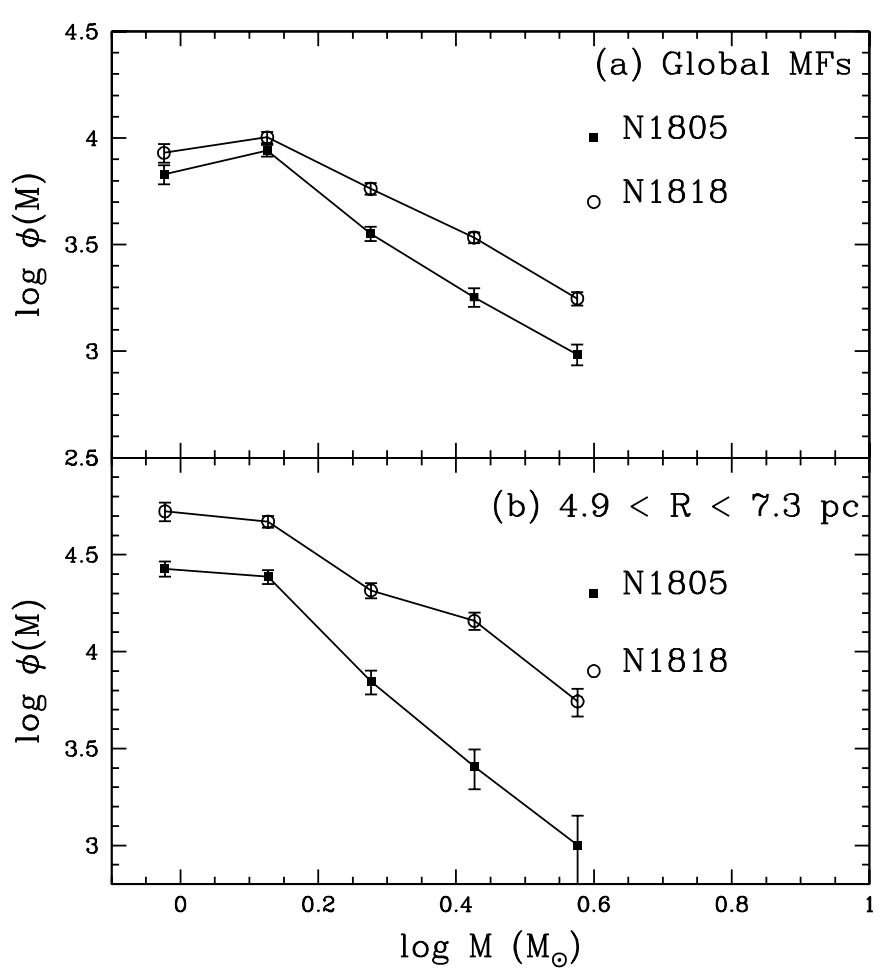

Fig. 24. Comparison between the NGC 1805 and NGC 1818 mass functions. Panel a) The global mass functions, within the range $0 \leq R \leq 25 \mathrm{pc}$. Panel $\mathbf{b}$ ) The mass functions for an outer radius, as indicated

analyze their density and surface brightness profiles, derive structural parameters and investigate the dependence of their luminosity functions with position. The cluster sample was chosen to display large variations in age, metallicity and structural parameters (Elson et al. 1998a,b; Beaulieu et al. 2000).

We obtain half-light radii and central stellar densities directly from the clusters density and surface brightness profiles. The clusters studied here display variable profile shapes, sizes and central densities. Our star number surface density and surface brightness profiles, despite being constructed by adding the individual contribution of each star within the cluster sample, show a general agreement with profiles obtained with integral ground-based photometry.

The luminosity functions (within the range $0.5 \lesssim$ $\left.M_{V} \lesssim 5.5,3 \gtrsim M / M_{\odot} \gtrsim 0.9\right)$ of our clusters display a systematic dependence with distance from the cluster centre, in the sense of being steeper at larger radii. This mass segregation is seen even in the two youngest clusters in our sample: NGC 1805 and NGC 1818. The ages of these two clusters are $\tau \lesssim 40$ Myrs. Assuming a central velocity dispersion in these clusters of $\sigma_{0} \simeq 1 \mathrm{~km} \mathrm{~s}^{-1}$, the two-body relaxation time for $3 M_{\odot}$ stars at $r \simeq 2 \mathrm{pc}$ from the centre (mid-distance of the most central luminosity function ring used) would be $\gtrsim 100$ Myrs. Thus, at least for these clusters, these trends in luminosity function shape may be primordial, something which would have important implications for the physics of star formation within protocluster gas clouds. We converted their luminosity functions into mass functions and made a direct comparison between the two: we find that NGC 1805 has a systematically steeper mass function than NGC 1818, both globally and in the outer regions, where dynamical timescales are likely to be longer than 1 Gyr.

Systematic steepening in the luminosity function with radial distance is also observed in NGC 1831, NGC 1868, NGC 2209 and H14. Luminosity function shapes vary considerably from cluster to cluster or even from one region to another in the same cluster. The luminosity functions are in many cases not well described by a straight line in the $\log \Phi$ vs. $M_{555}$ plane (a power-law in luminosity). However, slopes derived from linear fits still usefully trace the more significant changes in luminosity function shape and in the relative proportions of high and low mass mainsequence stars.

Steepening in the luminosity function from the cluster centre outwards is expected due to mass segregation caused by energy equipartition. $N$-body modelling of cluster sized samples of point masses also reproduce this trend. It would be very interesting to directly compare model predictions with the observed positional dependent luminosity functions (or mass functions) in this work. Vesperini \& Heggie (1997) have modelled the evolution of the cluster stellar mass function, taking into account relaxation, stellar evolution and disk shocking and tidal effects due to the Galaxy. Their simulations reconstruct the evolution of the mass function slope for different parts of a cluster. Unfortunately, the mass range probed in their simulations does not coincide with ours, preventing a straight comparison between the predicted slope variations and the observed ones.

We compared our global mass function obtained for NGC 1818 with that of Hunter et al. (1997) and found excellent agreement between the two. Unfortunately, to our knowledge, this is the only deep enough mass function determination from other authors to allow a direct comparison.

We are currently analyzing the colour magnitude diagrams of our clusters using objective statistical tools in an attempt to extract from the data the most reliable values for cluster age and metallicity. These in turn will allow more reliable conversion of the clusters luminosity functions into mass functions, making it easier to compare with the results of dynamical modelling. Mass functions and binary fractions are also currently being obtained from these data, by statistically comparing observed CMDs and luminosity functions to model ones.

Acknowledgements. We are deeply indebted to the late Rebecca Elson, not only for the crucial role she played in planning and carrying out this project, but also for the great deal we learned from her. BXS wishes to thank the hospitality and support of the IoA, in Cambridge, UK.

\section{References}

Aarseth, S. J. 1999, PASP, 111, 1333 
Beaulieu, S. F., et al. 1998, New Views of the Magellanic Clouds, IAU Symp. 190., ed. Y.-H. Chu, N. Suntzeff, J. Hesser, \& D. Bohlender, Victoria, British Columbia, Canada, 460

Beaulieu, S. F., Gilmore, G. F., Johnson, R. A., et al. 2000, The Evolution of Galaxies on Cosmological Timescales, ASP Conf. Ser., ed. J. E. Beckman, \& T. J. Mahoney

Castro, R., Santiago, B., Gilmore, G., Beaulieu, S., \& Johnson, R. 2000, MNRAS, submitted

Chiosi, C., Bressan, A., Portinari, L., \& Tantalo, R. 1998, A\&A, 339, 355

Corsi, C., Buonanno, R., Fusi-Pecci, F., et al. 1994, MNRAS, 271,385

de Oliveira, M. R., Bica, E., \& Dottori, H. 2000, MNRAS, 311, 589

Elson, R. 1991, ApJS, 76, 185

Elson, R., Gilmore, G., Aarseth S., et al. 1998a, The Magellanic Clouds and Other Dwarf Galaxies, Proc. of the Bonn/Bochum-Graduiertenkolleg Worshop, ed. T. Richtler, \& J. M. Braun (Shaker Verlag, Aachen), 243

Elson, R., et al. 1998b, New Views of the Magellanic Clouds, IAU Symp. 190, ed. Y.-H. Chu, N. Suntzeff, J. Hesser, \& D. Bohlender, Victoria, British Columbia, Canada, 417

Elson, R., Sigurdsson, S., Davies, M., Hurley, J., \& Gilmore, G. 1998c, MNRAS, 300, 857

Ferraro, F., Fusi-Pecci, F., Testa, V., Greggio, L., et al. 1995, MNRAS, 272, 391

Flower, P. J., Geisler, D., Olszewski, E. W., \& Hodge, P. 1980, ApJ, 235, 769

Geisler, D., Bica, E., Dottori, H., Clariá, J., Piatti, A., \& Santos, J. 1997, AJ, 114, 1920

Goodwin, S. 1997a, MNRAS, 286, 669

Goodwin, S. 1997b, MNRAS, 286, L39

Heggie, D. C., \& Aarseth, S. J. 1992, MNRAS, 257, 513

Holtzman, J., et al. 1995a, PASP, 107, 156
Holtzman, J., et al. 1995b, PASP, 107, 1065

Hunter, D., Light, R., Holtzman, J., Lynds, R., \& O’Neil, E. 1997, ApJ, 478, 124

Johnson, R., Elson, R., Gilmore, G., Beaulieu, S., \& Tanvir, N. 1998, in NICMOS and the VLT: A New Era of High Resolution Near Infrared Imaging and Spectroscopy, ESO Conf. and Workshop Proc. 55, ed. W. Freudling, \& R. Hook, Pula, Sardinia, Italy

Johnson, R. A., Beaulieu, S. F., Gilmore, G. F., et al. 2000, MNRAS, submitted

Mateo, M. 1988, ApJ, 331, 261

Olsen, K., Hodge, P., Mateo, M., et al. 1998, MNRAS, 300, 665

Olszewski, E. W., Schommer, R. A., Suntzeff, N. B., \& Harris, H. C. 1991, AJ, 101, 515

Panagia, N., Gilmozzi, R., Macchetto, F., Adorf, H.-M., \& Kirshner, R. 1991, ApJ, 380, L23

Santiago, B. X., Elson, R. A. W., \& Gilmore, G. F. 1996, MNRAS, 281, 1363

Santiago, B. X., Elson, R. A. W., Sigurdsson, S., \& Gilmore, G. F. 1998, MNRAS, 295, 860

Scalo, J. 1998, in The Stellar Initial Mass Function, 38th Herstmonceaux Conference, ed. G. Gilmore, \& D. Howell, Astron, Soc. Pacific Conf. Ser., 142

Spurzem, R., \& Aarseth, S. J. 1996, MNRAS, 282, 19

Vallenari, A., Chiosi, C., Bertelli, G., Meylan, G., \& Ortolani, S. 1992, AJ, 104, 1100

Vallenari, A., Bomans, D. J., \& de Boer, K. S. 1993, A\&A, 268,137

Vesperini, E., \& Heggie, D. C. 1997, MNRAS, 289, 898

Westerlund, B. E. 1990, A\&AR, 2, 29

Whitmore, B., Heyer, I., \& Casertano, S. 1999, PASP, 111, 1559

Will, J.-M., Bomans, D., \& De Boer, K. 1995, A\&A, 295, 54

Worthey, G. 1999, priv. comm. 